Fernández Palop, M.P. \& Caballero García, P.A. (2017). El libro de texto como objeto de estudio y recurso didáctico para el aprendizaje: fortalezas y debilidades. Revista Electrónica Interuniversitaria de Formación del Profesorado, 20(1), 201-217.

\title{
El libro de texto como objeto de estudio y recurso didáctico para el aprendizaje: fortalezas y debilidades
}

\author{
M. Pilar Fernández Palop y Presentación A. Caballero García
}

Universidad Camilo José Cela

\section{Resumen}

Con el fin de abrir campo a futuras investigaciones sobre el libro de texto como recurso didáctico en el aula y su posible repercusión en el aprendizaje, el presente estudio parte de cómo ha sido entendido conceptualmente por distintos autores y cuáles son las características principales que lo definen. Dado que se trata del instrumento más utilizado para la enseñanza y el aprendizaje de distintas materias, se reflexiona sobre sus ventajas y sus limitaciones como recurso didáctico, y se muestran evidencias de cómo en ocasiones, lejos de ser fuente de conocimientos, puede ser un obstáculo para el aprendizaje, tal y como se refleja en distintos estudios que abordan los libros de texto, y en particular, cuando se trata del libro de texto de matemáticas. Por último, en la búsqueda de posibles causas del bajo rendimiento de los alumnos españoles en matemáticas, y de la mejora en la didáctica de la matemática, se hace una propuesta de investigación futura, cuyo objeto de estudio sean los libros de texto de Matemáticas.

\section{Palabras clave}

Libros de texto; recursos didácticos; matemática; errores matemáticos.

Contacto:

Presentación A. Caballero García. Facultad de Educación. Universidad Camilo José Cela. C/ Castillo de Alarcón, 49 • Urb. Villafranca del Castillo · 28692 Madrid. Email: pcaballero@ucjc.edu 


\title{
The textbook as an object of study and teaching resource for learning: strengths and weaknesses
}

\begin{abstract}
Looking for new future researches based on the textbook as a teaching resource in the classroom and its possible impact on learning, this study begins describing how it has been understood by different authors and what the main characteristics that define it are. As it's an instrument mostly used for teaching and learning different subjects, we reflect its advantages and its limitations as a teaching resource, and we show evidence of how sometimes, far from being a source of knowledge, it can be an obstacle to learning, as it is reflected in various studies focused in textbooks, particularly in Mathematics textbook. Finally, seeking causes of the low Spanish students in mathematics performance, and improvement of mathematics teaching, we make a proposal for future researches centered in mathematics textbooks.
\end{abstract}

\section{Key words}

Textbooks; didactic resources; mathematics; mathematic error.

\section{INTRODUCCIÓN. El libro de texto: significado y sentido}

A pesar de la gran variedad de recursos educativos existentes en el mercado y los avances producidos en el campo tecnológico, la práctica de la enseñanza se sigue apoyando mayoritariamente en el libro de texto (Cabero, Duarte, \& Barroso, 1989; García Mateos \& Caballero García, 2005). Los libros de texto tienen un uso muy extendido en el aula (Parcerisa Arán, 1996), se utilizan por profesores y alumnos como un instrumento al servicio del aprendizaje. Ahora bien, ¿ha sido siempre conceptualizado de la misma manera? ¿Ha afectado eso a su funcionalidad y uso? ¿Qué consecuencias ha tenido en los resultados académicos? El presente artículo intentará dar respuesta a cada uno de estos interrogantes?

Existen multitud de definiciones para el libro de texto, algunas más precisas y otras más globales. Richaudeau (1981) define el libro de texto como "un material impreso, estructurado, destinado a utilizarse en un determinado proceso de aprendizaje y formación" (p. 51). Torres Santomé (1994) indica que los libros de texto son aquéllos que están diseñados para su uso solamente en aulas y centros de enseñanza, y que "contienen la información que los alumnos y alumnas precisan para poder demostrar que cumplen los requisitos para aprobar una determinada asignatura" (p. 155).

Los libros de texto, como portadores del conocimiento y la información que una generación desea transmitir a la siguiente, con frecuencia se encuentran en el centro de la controversia política (The Georg Eckert Institute for International Textbook Research, 2016).

Las leyes educativas en España han pasado de prescribir los libros que se deben usar en el aula (Ley $\mathrm{N}^{\circ} 14,1970$ ), previa autorización expresa a las editoriales, a la confección del currículo por parte del profesor de acuerdo a unas pautas determinadas por las administraciones educativas (las Comunidades Autónomas) y a una revisión a posteriori de los materiales producidos por las editoriales (Ley No 10, 2002; Ley Orgánica № 2, 2006; Ley Orgánica ํ8, 2013). 
En la práctica no es el profesor el que diseña el currículo (Cintas Serrano, 2000), sino que deja esa tarea a las editoriales, de cuya concreción curricular se sirven para ejercer su labor en el aula. De este modo, el libro es una guía que dirige el curso de la enseñanza en buena parte de las aulas. A pesar de las reformas educativas que han tenido lugar en los últimos años, esta práctica sigue siendo generalizada en nuestras aulas. Esta circunstancia supedita la metodología de enseñanza a la actividad comercial. Un asunto nada baladí, teniendo en cuenta que, según un estudio de la Universidad de Santiago de Compostela (Varela Mallou, 2008), el sector editorial genera anualmente un $0,5 \%$ del PIB europeo, siendo este porcentaje mayor en el caso de España (0,7\%). Además, el peso de los libros de texto fue de un $25,7 \%$ del total de ventas del sector editorial en 2007. El Informe sobre "El sector del libro en España 2013-2015" del Ministerio de Educación, Cultura y Deporte y el Observatorio de la Lectura y el Libro (2015) sostiene que la publicación de libros impresos subió un 1\% durante 2015 y los nuevos modelos de negocio favorecerán las ventas de los libros impresos que además aumentarán su valor debido a la proliferación de la venta de libros digitales.

En la misma línea, Choppin (1992) considera que "los manuales pueden ser estudiados desde distintos puntos de vista, ya que son a un tiempo producto de consumo, soporte de conocimientos escolares, vectores ideológicos y culturales e instrumentos pedagógicos" ( $p$. 102). Según esto, las distintas aproximaciones a una definición de libro de texto tendrían que llevarse a cabo desde alguna de estas perspectivas.

En cuanto a la influencia que el libro de texto tiene en el aula, "se estima que los libros de texto llegan a condicionar de manera importante el tipo de enseñanza que se realiza, ya que muchos enseñantes lo utilizan de manera cerrada, sometiéndose al currículum específico que se refleja en él, tanto en lo que se refiere a los contenidos de aprendizaje como a la manera de enseñarlos" (Parcerisa Aran, 1996, p. 35). Por tanto, no es que muchos libros de texto tengan determinadas características porque es lo mejor para los alumnos, sino porque es lo mejor para su propia perpetuidad.

"Ningún libro de texto, por bueno que sea, será un instrumento de validez universal; siempre habrá que emprender actividades adicionales de índole muy diversa" (Cockcroft, 1985 , p. 114). Y es que el libro de texto no puede sustituir al profesor en ningún caso, aunque la tendencia general sea que el libro dirija de algún modo la actuación del docente.

Es, pues, justo otorgar al libro de texto, en base a su uso en el aula y a las propiedades con que ha sido concebido, un poder extraordinario en el devenir educativo de los centros que lo utilizan, aunque eso pueda suponer un lastre para la investigación y puesta en práctica de nuevas metodologías educativas.

Sin embargo, aunque no es común debido a la confianza que normalmente se deposita en ellos, sí es legítimo preguntarse ¿es siempre correcto el contenido que aparece en los libros de texto de Matemáticas?

Sin entrar en las connotaciones y en el debate que pudiera generar el libro de texto como producto de consumo, como soporte de conocimientos e instrumento pedagógico -porque no es objeto del presente artículo-, en nuestra aproximación a la definición de libro de texto, nos acogemos a la distinción que Johnsen (1996) hace entre libro de texto y libro escolar, según su intención de ser usados en secuencias de enseñanza (libro de texto) o no (libro escolar). En su opinión, el término libro de texto "queda reservado para libros escritos, diseñados y producidos específicamente para su uso en la enseñanza" (p. 25), mientras que el término libro escolar se utilizaría para "libros empleados en la enseñanza, pero menos íntimamente ligados a las secuencias pedagógicas" (p. 26). El término libro escolar ("schoolbook") aparece por primera vez en inglés en la década de 1750, y más 
habitualmente en la de 1770. El término libro de texto ("textbook") no aparece en inglés hasta la década de 1830.

De igual modo, y desde las mismas perspectivas, Marguerite Puget (1963) distingue entre manual (libro de texto) y libro escolar:

El manual (...) es un libro que expone las nociones esenciales de una disciplina dada, en un nivel dado. Se corresponde a un curso, se dirige a una clase. Pero hay libros escolares que no son manuales: los diccionarios, los atlas, los resúmenes de recordación, todo simplemente porque su uso es estacionario sobre muchos años de la escolaridad, cuando no toda la escolaridad (p. 218).

La misma distinción (entre libro de texto y libro escolar) se mantiene en Hamilton (1990), que además incluye la perspectiva del libro de texto como vector ideológico y cultural. Según este autor, el libro de texto no es solo un libro utilizado en las escuelas, sino que se trata de un libro que ha sido conscientemente diseñado y organizado para servir a los fines de la escolarización. En este sentido, los libros de texto están orgánicamente unidos a las circunstancias cambiantes de la escolarización, y debería ser posible "leer" las formas de la escolarización a partir de los libros de texto que las acompañan.

Choppin (2000) destaca la relación que existe entre el libro de texto y las circunstancias del momento en el que es editado:

El manual [libro de texto] se presenta como el soporte, el depositario de los conocimientos y de las técnicas que en un momento dado una sociedad cree oportuno que la juventud debe adquirir para la perpetuación de sus valores. Los programas oficiales, cuando existen, constituyen la estructura sobre la cual los manuales deben conformarse estrictamente (p. 108).

Cabero, Duarte y Romero (1995) señalan las siguientes características básicas de los libros de texto, las cuales los diferencian respecto a otros materiales impresos utilizados en el sistema escolar:

- es un instrumento destinado a la enseñanza e instrucción con un fuerte sentido escolar,

- incluye teóricamente la información que debe ser procesada por el estudiante en un período de tiempo reglado,

- posee una configuración de acuerdo a pautas de diseño específicas, que persiguen presentar la información de una manera sistemática, de acuerdo a principios didácticos y psicológicos que faciliten la comprensión, dominio y recuerdo de la información por parte del estudiante, y

- tiende a compartimentalizar los contenidos, tanto diacrónicamente como sincrónicamente.

En un intento de aportar una descripción más completa del concepto de libro de texto, Moya Pardo (2008) recoge una síntesis de la caracterización del libro de texto a partir de lo que afirma Venegas (1993) respecto de lo que puede considerarse libro de texto o no. En su opinión, el libro de texto es un instrumento básico en el proceso de enseñanza y aprendizaje, mediador del conocimiento, que permite al niño una autonomía en su trabajo, proporcionándole ejercicios, e induciéndole a llevar a cabo experimentos. Además, señala que el libro debe partir de las experiencias del niño y estar vinculado a los avances e innovaciones que se produzcan en el campo de la ciencia. Por último, el libro constituye una unidad en la que se incluyen los distintos elementos para la incorporación por parte del 
alumno de los contenidos del currículo. Y, como factor determinante de lo que "no es un libro de texto", subraya que no debe ser un sustituto del profesor en su labor docente.

Otra descripción es la que llevan a cabo Alzate, Gómez y Romero (1999), según los cuales, el libro de texto "es un conjunto de hojas impresas que guardan relación con las actividades que se llevan a cabo en las escuelas, (... ) constituye un instrumento que les ofrece al educador y al estudiante una información sobre los diversos temas, o desarrollos que las disciplinas científicas tienen en un momento dado" (p. 28-29). Estos autores distinguen los libros de texto de los libros de consulta (libro escolar), afirmando que estos últimos pueden ser utilizados en las escuelas para ampliar información acerca de un tema del currículo, mientras que el contenido del libro de texto está expresamente adecuado a la transmisión del currículo, y esto tiene repercusión en todos los ámbitos del libro: en su estructura, diseño, etc.

Teniendo en cuenta estas consideraciones, en nuestras reflexiones sobre el libro de texto de matemáticas y sus repercusiones para los procesos de enseñanza-aprendizaje, utilizaremos la siguiente definición: "libro, ya sea impreso en papel o en cualquier otro formato, editado con el fin de servir de guía para enseñar Matemáticas en un determinado curso/nivel educativo perteneciente a un plan de estudios".

\section{Argumentos a favor y en contra del libro de texto como recurso didáctico}

Como hemos visto, los libros de texto son un instrumento en el proceso de enseñanzaaprendizaje. Ahora bien ¿lo potencian o, por el contrario, lo dificultan? En la respuesta a este interrogante puede considerarse que sus efectos son positivos o negativos, tanto para la enseñanza como para el aprendizaje de una determinada materia, pero como indica Prendes (1994) nosotros nos posicionamos en la idea de que, independientemente de esta connotación, "el mal no está en el medio en sí, sino en el uso que de él se haga. El libro puede contribuir a la reflexión, a la creación y al aprendizaje innovador o por el contrario puede convertirse en instrumento que degrada y deforma la enseñanza" (p. 428).

Cabero et al. (1995) señalan que, entre los argumentos que utilizan los detractores del libro de texto está el hecho de que en los textos se escoge los contenidos que aparecen, eliminando otros, pudiendo ser los motivos de dicha elección políticos o ideológicos. Además, en los libros no existe una confrontación de los contenidos con la realidad, reduciendo así la búsqueda y la experimentación por parte del alumno, y mermando su espíritu crítico. En ocasiones, el libro de texto sustituye al profesor, que se convierte en un mero transmisor de lo que aparece en el libro, limitando así el desarrollo de metodologías que favorecen el aprendizaje, ya que, prácticamente, el único modo de aprendizaje que se propone a través de los libros es a través de la repetición y la memoria.

Alzate Piedrahita (1999), en un estudio posterior en el que se describen los argumentos de los partidarios y los detractores del libro de texto como instrumento pedagógico, se suma a lo indicado por Cabero et al. (1995), señalando que los detractores del libro consideran que el libro, al dar todo hecho al alumno, le priva de su capacidad de investigadora y crítica. Asimismo, al resaltar un determinado contenido, anula otros. $Y$ esto tiene consecuencias, ya que es el libro o más bien quien edita sus contenidos el que decide qué conocimientos son los que se deben ir transmitiendo a las siguientes generaciones. De igual forma, en cuanto a los argumentos en contra del libro de texto, estaría el hecho de que están escritos para los maestros, que son quienes los van a escoger, y no para los alumnos. Por otro lado, el libro de texto impone al maestro, y en consecuencia al alumno, el ritmo y la progresión que se ha 
de seguir. Además, no puede olvidarse que el manual es un producto comercial, y pueden ser las consideraciones de esta índole las que presidan su concepción y su difusión.

Entre los argumentos que destacan los partidarios del libro de texto, continua señalando Alzate, está que el manual sería garante de la igualdad de oportunidades. Al ser un material idéntico que tienen todos los alumnos, permite que aquellos que van más atrasados, o que por motivos de enfermedad no han podido acudir a clase (o, sencillamente, se han distraído durante la explicación), dispongan de aquellos contenidos que han sido explicados por el profesor, de forma que, a través de su trabajo autónomo fuera del aula, puedan mantener el ritmo del grupo. Por otro lado, los partidarios del texto escolar afirman que el libro haría posible la enseñanza, ya que permite al maestro centrarse en los alumnos que tuvieran una mayor dificultad, mientras sus compañeros llevan a cabo una actividad común. Por último, la autora subraya que el texto escolar asegura la relación entre la escuela y la familia, ya que permite a los padres conocer qué hacen sus hijos en clase, ayudarles en sus tareas o repasar sus lecciones.

Por su parte, Campanario (2001), al describir una serie de usos del libro de texto que pueden resultar innovadores, destaca, como los más frecuentes, que pueden ser: a) Fuente de información para alumnos y profesores; b) fuente de ejercicios y tareas de clase; y c) fuente de preguntas y ejercicios de evaluación.

Estos usos, que favorecen la labor educativa por un lado, también pueden tener su aspecto negativo, tal y como señalan Cabero et al. (1995), al describir cómo el libro de texto puede convertirse en elemento orientador de la actividad profesional del profesor o incluso puede devaluar su prestigio. Si la labor del maestro se limita a transmitir los contenidos que aparecen en el libro, en cierto modo su cualificación profesional en este desempeño podría ser mínima: bastaría con tener una buena educación en el trato con los alumnos y unas buenas dotes como reproductor de textos. Además, esa dinámica de seguir el libro en su sentido más estricto, haría de él un sujeto aislado, ya que no necesitaría de la relación con sus compañeros para resolver los problemas que le surgieran. En realidad, el responsable del rendimiento en el aula y de los resultados de la enseñanza no sería, por tanto, el profesor, sino el libro de texto.

Investigadores que se han dedicado al estudio del currículum y los textos desde distintas perspectivas han planteado en las últimas décadas controvertidas discusiones en torno a los mismos. Romero (2011) haciendo una exhaustiva revisión de las mismas nos resume que los cuestionamientos se han pronunciado sobre las distorsiones ideológicas, las limitaciones didácticas y los intereses comerciales subyacentes de los textos escolares; también sobre el control técnico que ha primado en la escuela a través de los textos escolares y la consecuente descalificación de los docentes, conduciendo a una pérdida de posibilidades de desarrollo de capacidades profesionales y, en algunos casos, a una cómoda y flexible adscripción por parte de los docentes. Desde una posición alternativa se han realizado estudios que nos testimonia Gimeno Sacristán en 1997, sobre la política cultural y la influencia del mercado de los libros de texto en las reformas educativas, que nos advierten de la necesidad de contemplar la realidad institucional de las escuelas y del trabajo docente, que hace de los materiales de enseñanza una ayuda necesaria en el cotidiano escolar. El desarrollo de la multimedia y los nuevos lenguajes suman discusiones en torno a la presencia y uso del libro en la escuela, a la amenaza de desaparición del libro en papel, con el aumento en el uso del e-book.

Los nuevos escenarios, sigue argumentando Romero, ponen de relieve más que nunca dos facetas del libro, por un lado, es un material escrito, diseñado y producido específicamente para su uso en la enseñanza en el marco de la escuela. Por otro lado, se trata de un 
complejo producto cultural influido por los nuevos enfoques didácticos, los cambios culturales y los flujos comunicacionales, las políticas estatales de dotación de libros a las escuelas, las lógicas del mercado y el consumo, entre otros. En su opinión, estas dos caras del libro influyen en los sentidos, no necesariamente contradictorios, que se le asignan al producirlo y usarlo: como instrumento pedagógico y como mercancía. Asimismo, como herramienta destinada al aprendizaje, los textos escolares son los únicos libros que en la mayoría de las instituciones educativas se convierten en obligatorios. Con frecuencia se trata del primer libro al que acceden los alumnos. Debido a esto, hay voces que argumentan que el texto escolar es un instrumento de democratización.

Hasta los años setenta, su campo de estudio estuvo circunscrito a obras individuales referidas a la historia y al contenido de los libros. Durante las décadas siguientes, la investigación sistemática aumentó considerablemente dando lugar a una vasta bibliografía que da cuenta de las definiciones, dimensiones y campos de investigación que conforman el universo de los textos escolares. En la actualidad existen ámbitos de estudio, investigación y circulación de saberes como son los centros de investigación especializados en varios países: la Asociación Internacional para la Investigación de los Libros de Texto y los Medios de Comunicación Educativos (IARTEM), Oslo (Noruega); el Instituto George Eckert para la Investigación Internacional en Textos Escolares, Braunschweig (Alemania); el Instituto de Investigación de Libro de Texto, Viena (Austria); el Instituto para la Investigación del Texto Educativo, Härnösand (Suecia); el Centro de Investigaciones del Libro de Texto, Tokyo (Japón) y el Centro de Investigación Manes (Manuales Escolares), Madrid (España). Los autores y editores, en el deseo de responder a las recomendaciones de las investigaciones y a los criterios de selección de los Comités de aprobación, tienden a ser consecuentes antes que inventivos (Romero, 2011).

Gran parte de las investigaciones referidas a los libros escolares se han centrado en el análisis de su contenido ideológico. Estos estudios han contribuido en gran medida a revisar los distintos modos en que se filtran en los textos concepciones y estereotipos legitimadores de desigualdad. Sin embargo, se ha demostrado que el libro de texto en sí mismo no posee un poder supremo sino que la diversidad de lecturas que realizan los docentes y estudiantes en cada contexto específico genera resistencias y cuestionamientos.

En la investigación más actual se abren perspectivas que intentan relacionar el libro de texto con el contexto social en el que es producido y utilizado. En esta dirección se intentó ampliar el campo de estudio a partir de la introducción de problemas tales como las formas literarias y las modalidades de uso en el aula, la producción y el mercado de los textos escolares, la selección y distribución de los libros

Pero es recién en los últimos tiempos que se evidencia un crecimiento de investigaciones sobre el uso de los textos escolares en el aula. El término "Textbook Pedagogy" introducido por Lambert en 2002 y por Horsley y Walker en 2003, se refiere a la forma en que los profesores utilizan los libros en clases, cómo acceden a ellos y cómo los adaptan y crean un contexto para su uso. El término ha sido utilizado, además, para dar cuenta de aquellas funciones y características de los textos que pueden mejorar o restringir el aprendizaje, en tanto influyen sobre el uso que el profesor hace del libro de texto en el aula. Se trata de una serie de estudios que, en general, asumen, una perspectiva psicológica. Los resultados dan cuenta de la variedad de formas en que los maestros seleccionan y utilizan los libros con sus alumnos. El texto escolar define y desarrolla una serie de temas que son subvertidos y alterados por las prácticas docentes cuando utilizan el libro. Si entendemos que a partir del uso, el libro se convierte en un nuevo texto, el resultado es una variedad inmensa de articulaciones, omisiones y agregados no incluidos en la producción de origen 
por los editores. Las razones más comunes para esas transformaciones se asocian al cumplimiento de las exigencias curriculares en el tiempo escolar disponible, a convertir las propuestas del libro en algo más atractivo y comprensible para los alumnos y a ofrecer "lo propio" en el sentido de un aporte personal y original del docente a sus alumnos. Recientes y escasas investigaciones acerca de los usos de los textos escolares como las de Bauer en 2009; Bryce en 2011; Duarte en 2010; y Ray Bazán en 2011, entre otros, ofrecen nuevas entradas de indagación incorporando la perspectiva de los estudiantes a la hora de usar los textos escolares y otros materiales educativos generalmente vinculados con las nuevas tecnologías. Estos estudios demuestran que, en los modos en que los textos escolares son usados, se relacionan heterogéneas redes de actividad en las que personas, prácticas, saberes y objetos están estrechamente conectados.

En suma, opinamos como Romero (2011), que atendiendo al estado actual de las investigaciones sobre textos escolares, ya no resulta suficiente abordar el estudio de los manuales en el sentido de "objetos autónomos e independientes", como ha sido tradición en el campo de la manualística, sino que es menester considerarlos en su relación con los alumnos, los docentes y las familias, en función de la disciplina que se trate, las interacciones con otros artefactos culturales y su propio contexto de producción editorial.

Todavía se sabe poco acerca de los procesos de decisión de editores, de cómo el diseño del libro dirige la relación alumno-libro de texto. Cómo lo hacen y cómo su mediación es influida por este recurso didáctico está abierto a debate. Apoyamos la necesidad de investigación en este sentido.

\section{El libro de texto, ¿obstáculo para el aprendizaje?}

¿Es posible que un libro de texto concreto pueda ser un obstáculo para el aprendizaje más que una ayuda? Como indica Johnsen (1996), "los malos textos educativos son una verdadera calamidad para cualquier nación; los buenos textos, en cambio, producen un beneficio incalculable y constituyen una inversión en nuestro futuro internacional" (p. 15).

La contrapartida al libro de texto como fuente de información es la que recoge Orlans (1998, p. 11), rescatando una anécdota sucedida en la Universidad Estatal de Arizona, en la que cuenta que, poco después de que Hugh Trevor-Roper hubiese demostrado que los escritos de Sir Edmund Backhouse eran fraudulentos, un estudiante presentó su trabajo de fin de carrera sobre historia china basándose en dos libros de dicho autor. Cuando el trabajo recibió una "D" (suspenso), el estudiante protestó. Y el instructor le explicó que había utilizado sólo dos fuentes, y que ambas eran fraudulentas.

“Qué quiere decir con 'fraudulento’?”, preguntó el estudiante.

"Acaban de poner patas arriba los escritos de Backhouse, no es cierto".

“¿Qué quiere decir con 'no es cierto’?”, respondió el estudiante. "Tiene que ser cierto. Está en la biblioteca".

Para aquellos que no tienen desarrollado un espíritu crítico y no realizan una verificación de la información antes de emitir un juicio, para los que no tienen formación o la tienen insuficiente, el que una información esté en letra impresa en un volumen de una biblioteca le da garantía de verdad. Y esto, que en estudiantes universitarios es un peligro, se hace aún más acuciante en los escolares, que por su falta de madurez son más influenciables. Por esta razón, Aparicio (2000) sugiere a los docentes que fomenten en los alumnos el uso de varias fuentes de consulta y la motivación por una buena preparación. 
El hecho de que los libros de texto, en concreto los matemáticos o científicos -que son nuestro objeto de atención en este artículo-, presenten deficiencias o errores puede suponer un obstáculo para el aprendizaje. Dos expertos en educación, Daniels y Zemelman (2004), realizan una descripción del problema que encuentran en los libros de texto que utilizan los estudiantes americanos. En ella van enumerando una serie de características que hacen del libro de texto un obstáculo para el aprendizaje más que una ayuda. Según estos expertos, los libros de texto entrarían en la categoría de manual de referencia, como pueden serlo las enciclopedias o los diccionarios, que no siguen un discurso coherente que permita su lectura continuada, y esto los hace ilegibles. Además, citando a Roseman, Kulm y Shuttleworth (2001), afirman:

Hoy los libros de texto abarcan demasiados temas sin desarrollar ninguno de ellos en condiciones. Los conceptos centrales no se cubren con la suficiente profundidad como para dar al estudiante la oportunidad de comprenderlos verdaderamente. Mientras que muchos libros presentan las ideas clave que se describen en los estándares, pocos libros ayudan a los estudiantes a aprender esas ideas o a los profesores a enseñarlas correctamente (p. 56).

Al mismo tiempo, destacan que el empeño de llenar de imágenes los libros de texto, al estilo de los juegos de ordenador o las consolas que tanto atraen a los niños, ha decaído en un caos visual que impide su comprensión. $Y$ a esto hay que sumar la imprecisión con la que están escritos. En relación con este punto, el estudio recoge un trabajo publicado en Physics Today (Hubisz, 2001), en el que un equipo de investigadores catalogó más de quinientas páginas de errores encontrados en los 12 libros de texto de física más populares utilizados en las escuelas de enseñanza media de los Estados Unidos, algo que está en contraposición con un nivel de precisión aceptable. Por último, los autores subrayan, como Aparicio (2000) el hecho de que ofrecer al estudiante una única fuente de conocimiento merma su capacidad de crítica y le obliga a aceptar el texto como una única autoridad.

Esto último es lo que destaca el trabajo realizado por Gauld (1997), en el que se afirma que los libros de texto son la principal fuente de información para la adquisición de conceptos científicos en la mayoría de los estudiantes. Sin embargo, analizando una serie de libros de texto, el autor encuentra una serie de errores que aparecen con frecuencia en dichos libros que, en consecuencia, se transmiten a profesores y estudiantes. $Y$ estos errores pueden llevar a conceptos erróneos generalizados.

Esta misma línea sigue argumentando una investigación realizada en los EE.UU. (Abraham, Grzybowski, Renner, \& Marek, 1992), en la que los autores se plantearon tres cuestiones acerca de cinco conceptos de química que aparecían en los libros de texto de alumnos de octavo grado. Las preguntas que se formularon fueron: “a) ¿Qué ideas falsas adquieren los alumnos de los libros de texto con relación a esos cinco libros de química? b) ¿Cómo es la capacidad de razonamiento de los alumnos en la relación entre esos conceptos y otros conceptos de química? c) ¿Cómo son de efectivos los libros de texto en la enseñanza de la comprensión de esos conceptos de química?". Para realizar el estudio, se realizaron dos tipos de pruebas. Unas se encaminaron a examinar el grado de comprensión de los conceptos de química e identificar conceptos erróneos. Otras iban dirigidas a evaluar el nivel intelectual de los estudiantes. $Y$, aunque el número de estudiantes evaluados (247) no permitía obtener resultados concluyentes, el estudio del nivel de comprensión de los cinco conceptos de química y de la naturaleza de los conceptos erróneos que tenían los estudiantes, indicaba un fallo general en los libros de texto para enseñar los conceptos de química, de forma que se permitiera una comprensión razonable. 
Otro estudio en el que se analiza el libro de texto como transmisor del conocimiento científico es el llevado a cabo por Kimberley, Norris, Phillips y Clarck (2003). En él se establece una comparación entre el contenido de una serie de libros de texto de ciencias y otra de reportajes divulgativos, sobre la base de cuatro características del análisis del discurso: tipo de texto, estado de verdad de los enunciados, uso de meta-lenguaje, y estatus científico en cuanto al razonamiento científico que utilizan. Los resultados resaltan que, tanto los libros de texto como los reportajes utilizan un tipo de texto en su mayor parte expositivo. En ninguno de los libros de texto había algún tipo de argumentación científica de ciencias, en contraposición con algunos de los reportajes, que sí contaban con una argumentación considerable. Sin embargo, en cuanto al hecho de presentar los contenidos como verdades definitivas, prácticamente todos los libros de texto los presentaban como tales, en contraposición con lo que sucedía en los reportajes, donde sólo dos terceras partes de las declaraciones fueron presentadas como verdades definitivas. Respecto del uso del meta-lenguaje, en los libros de texto de ciencias de secundaria resultaba difícil encontrar signos en los que se reflejara el método científico, tales como la observación o las relaciones de causa. En los reportajes divulgativos, estos signos eran más frecuentes (el triple de la hallada en los libros de texto). Por último, tanto los libros de texto como los reportajes se limitaban a recoger hechos o conclusiones, algo que reduce lo que es el verdadero aprendizaje de la ciencia.

Centrado ya en cómo el libro de texto favorece o no el razonamiento científico, encontramos otro estudio (Stern \& Roseman, 2004) en el que se observa el modo en el que nueve libros de texto de gran difusión tratan dos conceptos fundamentales de ciencias que aparecen en el currículo: la fotosíntesis y la respiración celular. En él se extrae que, aunque estos temas les han sido enseñados a los alumnos durante muchos años, la investigación sobre su aprendizaje indica que los estudiantes tienen dificultades en el aprendizaje de estos conceptos. De acuerdo con sus hallazgos, los materiales curriculares disponibles ofrecían poco apoyo para la consecución de las ideas clave elegidas para su estudio. En general, estos materiales no tenían en cuenta los conocimientos previos de los estudiantes, adolecían de representaciones que pudieran aclarar ideas abstractas, y eran deficientes en la explicación de fenómenos que perfectamente podrían ser comprendidos por los estudiantes.

Ahondando en la necesidad de transmitir no sólo hallazgos científicos sino también el método científico, en Victoria, Australia, se examinó (Wilkinson, 1999) el contenido de los libros de texto utilizados en el estudio de la física entre los años 1967 y 1997, centrándose en los siguientes aspectos: a) la ciencia como un conjunto de conocimientos, b) la ciencia como una manera de investigar, c) la ciencia como una forma de pensar, y d) la interacción entre la ciencia, la tecnología y la sociedad. Estos temas fueron escogidos porque aparecían en los objetivos del vigente Certificado de Educación de Victoria para el estudio de la física. Entre los resultados obtenidos, se vio que la mayoría de los textos analizados acumulaban un vasto conjunto de conocimientos científicos, sin embargo, ponían poco énfasis en la ciencia como una forma de investigar, y muy poco énfasis en la ciencia como una forma de pensar.

De hecho, los propios alumnos, también pueden percibir estas carencias, tal y como refleja la encuesta realizada a estudiantes universitarios (Besser, Stone, \& Nan, 1999) sobre la percepción que tenían de sus libros de texto. El $60 \%$ de ellos manifestó que no resultaban útiles para el aprendizaje. Algo semejante sugiere el estudio de Çokadar y Şahin (2009), y en este caso son futuros profesores los que expresan sus experiencias en el uso de los libros de texto. 
Como puede verse, este tipo de situaciones suponen un obstáculo didáctico (Brousseau, 1991), ya que constituyen un impedimento para el aprendizaje, debido a una elección que se hace en la enseñanza.

Si hacemos un repaso por estudios realizados específicamente sobre libros de texto de matemáticas, estos obstáculos son advertidos por diversos autores (Arcavi, 2006; Kline, 1973), tanto por el modo de transmitir los conceptos matemáticos, como porque el contexto en el que se presentan es un mero disfraz, como si dichos contenidos matemáticos no fueran una respuesta real a situaciones reales.

Fernández Bravo (2010) hace hincapié en los enunciados de los problemas, tanto en los que aparecen en los libros de texto de matemáticas, como en los que proponen los profesores o crean los propios niños, e indica las consecuencias que pueden tener para el aprendizaje el trabajar con problemas mal enunciados.

Cuando se presenta en el aula la redacción de una situación problemática, el alumno toma como modelo lingüístico lo que se expresa, lo retiene y lo asocia posteriormente con el contenido resolutorio de la situación. El rigor, la precisión y la claridad del lenguaje que se presenta al alumno son de exagerada importancia. Los problemas que se leen a lo largo de la actividad escolar son muchos. $Y$ son muchos los que contienen incorrecciones semánticas, sintácticas y matemáticas en sus enunciados. Se lee, tanto lo que está bien como lo que está mal redactado, y las interpretaciones no se sujetan tanto al sentido de su expresión, sino a la intuición de ese sentido. La fijación de ideas claras no puede tener un carácter fragmentario, sino un carácter sistémico, integral. Hay que elevar a una magnitud prioritaria la correcta formulación verbal del problema, poniendo excesivo cuidado en la presentación de su información (p. 42).

Comparando los libros de texto de distintos países, Haggarty y Pepin (2002) estudiaron el tratamiento del concepto de ángulo en los libros de texto más populares de Inglaterra, Francia y Alemania. El análisis de los datos sugiere que a los estudiantes en los diferentes países se les dan distintas matemáticas y difieren en las posibilidades de aprender matemáticas, las cuales se ven influidas por los libros de texto y sus maestros.

En ocasiones, se han llevado a cabo revisiones de libros de texto de matemáticas. El premio Nobel de física Feynman (1987), al describir su experiencia en este sentido, relata lo encontrado en un libro en el que se decía que la matemática estaba relacionada con la ciencia, y, a modo de ejemplo, se proporcionaba la temperatura aproximada de diversas estrellas, y se pedía a los niños hallar la temperatura global de un conjunto de estrellas.

$Y$ yo reviento horrorizado (...). ¡El absurdo perpetuo! Hallar la temperatura total de dos estrellas es algo falto por completo de sentido. ¡Nadie suma la temperatura de las estrellas, salvo tal vez para calcular la temperatura media de un grupo de estrellas, pero jamás para hallar la temperatura total! ¡Era horrible! Todo era una historieta para hacer sumar al niño; los autores no tenían ni idea de lo que hablaban. Era como ir leyendo frases con unos cuantos errores tipográficos, y entonces, de pronto, aparece una frase entera escrita al revés, de fin a principio. Así eran las matemáticas. ¡No había remedio! (p. 340-341).

Sin embargo, Feynman no es el único que describe errores en libros de texto de matemáticas. En la literatura científica es posible encontrar, no muchos, pero sí otros autores que han identificado y demostrado la existencia de errores en libros de texto de matemáticas (Brewer, 1986; Fernández Palop y Caballero García, 2013; Fernández Palop, Caballero García, \& Fernández Bravo, 2013; Jaime Pastor, Chapa Aguilera, \& Gutiérrez 
Rodríguez, 1992; Kolmogorov, 1946; Muntean, 2011) que pueden ser un obstáculo para el aprendizaje y un elemento explicativo, entre otros, de los malos resultados del aprendizaje.

\section{Discusión, conclusiones y prospectiva}

En la segunda mitad del siglo XX, han ido surgiendo "diversas organizaciones internacionales que tienen como finalidad promover y desarrollar iniciativas internacionales de evaluación de los sistemas educativos para compararlos y proporcionar información para su mejora" (López Varona \& Moreno Martínez, 1997, p. 5). Entre estas organizaciones destacan tanto la International Association for the Evaluation of Educational Achievement (IEA), como la Organización para la Cooperación y el Desarrollo Económicos (OCDE), que han elaborado programas para la evaluación de alumnos en las competencias de lectura, matemáticas y ciencias.

A lo largo de estos años, España ha participado en varios de estos programas para evaluar la competencia matemática de sus alumnos: el organizado por la IEA, el Trends in International Mathematics and Science Study (TIMSS), realizado en 1995 a alumnos de 13 años y, posteriormente, a alumnos de 10 años en 2011; y el que desarrolla la OCDE, el Programa para la Evaluación Internacional de Alumnos (PISA), en el que ha participado en sus ediciones de 2000, 2003 (en la que se examinó con mayor profundidad la competencia matemática), 2006, 2009 y 2012. En estas pruebas, además de las matemáticas, se han evaluado la lectura (PISA) y los aprendizajes las ciencias (TIMSS y PISA).

En todas estas pruebas, los alumnos españoles han quedado siempre por debajo de la media de los países participantes, lo que pone de manifiesto la importancia de buscar causas de este bajo rendimiento por parte de nuestros estudiantes.

Uno de los recursos didácticos más utilizados es el libro de texto. Según los datos del Instituto de Evaluación del Ministerio de Educación (2009), dicho recurso es utilizado por un 99,1\% de los alumnos de Educación Primaria, de ahí la importancia de desarrollar investigaciones que lo incluyan como objeto de estudio.

En primer lugar, podríamos preguntarnos si los libros de texto de nuestros alumnos favorecen la adquisición de las competencias analizadas por PISA o TIMSS en el ámbito de la matemática, como han hecho otros autores (Gallardo-Gil et al., 2010; Hatzinikita, Dimopoulos, \& Christidou, 2008) en las otras áreas evaluadas en PISA.

En nuestro artículo hemos partido de una definición de libro de texto, dado testimonio de posiciones a favor y en contra del libro de texto como recurso didáctico, revisado la escasa literatura existente sobre las deficiencias (errores) de los libros de texto de contenido científico, que han sido nuestro centro de interés y de análisis, y argumentado que hay autores que han encontrado errores matemáticos en libros de matemáticas. Si esto es así, se precisa mayor investigación sobre el libro de texto, en particular, sobre el libro de texto de matemáticas, para identificar si se ajusta o no a lo que dicta la matemática, esto es, si contiene o no errores matemáticos, y qué tipo de error contiene, su tipología, ubicación, y posibles repercusiones en la didáctica del profesorado, en los aprendizajes de nuestros alumnos, y en los resultados de los estudiantes españoles en la evaluación internacional.

Si centramos nuestro interés en los errores matemáticos en libros de texto, podríamos preguntarnos: ¿Son todos los errores iguales, o existen unos errores que son más graves que otros? ¿Podemos considerar de igual modo un libro que contenga un mismo error en dos secciones distintas que otro que contenga dos errores distintos? Estos factores ponen de manifiesto la posibilidad de elaborar y validar un instrumento que permita medir el nivel 
de error de un libro, de forma que se pueda valorar un libro de texto de matemáticas en lo que a errores matemáticos se refiere.

Por otro lado, también podríamos centrarnos en el estudio de errores didácticos (Brousseau, 1976, 1991; Escolano Vizcarra \& Gairín Sallán, 2005; Jaime Pastor et al., 1992). Dichos errores didácticos, en caso de identificarse, podrían afectar igualmente al aprendizaje de la matemática por parte de los alumnos, por lo que podría ser de interés para la ciencia, por un lado, verificar la existencia de dichos errores didácticos, y, por otro, presentar propuestas de cambio en las que se haya comprobado que el aprendizaje mejora.

Puede ser útil para estas propuestas el estudio de los libros de texto que se utilizan en Singapur (Ban Har, 2011; Ministry of Education, 2011), cuyos alumnos encabezan los resultados de las distintas pruebas internacionales llevadas a cabo (Beaton et al., 1996; López Varona \& Moreno Martínez, 1997; Ministerio de Educación, 2010; Ministerio de Educación Cultura y Deporte, 2012; Ministerio de Educación y Ciencia, 2004, 2007, 2008; Ministerio de Educación y Política Social y Deporte, 2005; Mullis, Martin, Foy, \& Arora, 2012; OECD, 2012), lo que puede ser una prueba de su eficacia. Son libros que están estructurados de forma que paulatinamente se puedan adquirir los conceptos matemáticos con profundidad. Utilizan un enfoque que comienza con representaciones pictóricas de los contenidos, para pasar más tarde a enfoques abstractos, haciendo uso de definiciones ostensivas, las cuales permiten la transmisión de los conceptos en los niveles inferiores, sin eliminar el debido rigor. Cada lección se inicia con una introducción al tema, que utiliza diagramas y modelos para ilustrar el concepto básico, seguido de tareas específicas de aprendizaje. Estos ejercicios utilizan diagramas adicionales e imágenes para mostrar diferentes formas de pensar sobre el concepto, y proporcionan ejemplos guiados para que los estudiantes practiquen y se familiaricen con el tema. Además, cuentan con libros de ejercicios en los que se incluyen una gran variedad de problemas, desde los que implican la adquisición de rutinas, hasta problemas matemáticos cada vez más complejos (Ginsburg, Leinwand, Anstrom, \& Pollock, 2005).

En relación con los libros, Muntean (2011) se plantea esta pregunta: “¿Por qué los errores de los libros de texto se parecen tanto?", y continúa: "Los autores de los libros de texto acuden a otros libros de texto, que a su vez se inspiran en otros y así sucesivamente, una práctica favorecedora de la reproducción de errores" (p. 51), sin embargo el autor no aporta ningún dato que avale dicha hipótesis.

Proponemos un estudio cuyo objetivo sea la búsqueda de evidencias que permitan aceptar o rechazar esta hipótesis y, en caso de aceptarla, estudiar las posibles consecuencias de esta práctica para el aprendizaje de nuestros alumnos.

La investigación en libros de texto es un campo relativamente nuevo y todavía no está representado como un tema en las universidades. Esta situación hace que sea aún más crucial para los que trabajan en el campo abordarla desde una variedad de disciplinas académicas y contextos regionales, la necesidad de recurrir a un espacio de discusión académica y apoyo mutuo y de proporcionar recursos para la provisión de datos y fuentes de información (The Georg Eckert Institute for International Textbook Research, 2016).

El estudio de los libros de texto como fuente de aprendizaje sigue siendo un apasionante mundo por descubrir y un enclave en la calidad de nuestro sistema educativo. 


\section{Referencias}

Abraham, M. R., Grzybowski, E. B., Renner, J. W., \& Marek, E. A. (1992). Understandings and misunderstandings of eighth graders of five chemistry concepts found in textbooks. Journal of Research in Science Teaching, 29(2), 105-120.

Alzate Piedrahita, M. V. (1999). El texto escolar como instrumento pedagógico: Partidarios y detractores. Revista de Ciencias Humanas, 21(Septiembre), 110-118.

Alzate Piedrahita, M. V., Gómez, M., \& Romero, F. (1999). Textos escolares y Representaciones Sociales de la familia. Definiciones, dimensiones y campo de investigación. Pereira: Universidad Tecnológica de Pereira.

Aparicio, F. (2000). Pautas para la mejora de la calidad en la enseñanza de estadística en ingeniería de telecomunicación. Relieve: Revista ELectrónica de Investigación y EValuación Educativa, 6(1). Consultado el 30 de mayo 2014, en http://www.uv.es/RELIEVE/v6n1/RELIEVEv6n1_2.htm

Arcavi, A. (2006). Lo cotidiano y lo académico en Matemáticas. Números, 63, 3-23.

Ban Har, Y. (2011). Primary Mathematics (Standards Edition) Textbook 6, A-B. Singapore: Marshall Cavendish Education.

Beaton, A. E., Mullis, I. V. S., Martin, M. O., González, E. J., Kelly, D. L., \& Smith, T. A. (1996). Mathematics Achievement in the Middle School Years: IEA'S Third International Mathematics and Science Study (TIMSS). Boston: Center for the Study of Testing, Evaluation, and Educational Policy, Boston Collage.

Besser, D., Stone, G., \& Nan, L. (1999). Textbooks and teaching: A lesson from students. Journalism and Mass Communication Educator, 53(4), 4-17.

Brewer, J. K. (1986). Behavioral statistics textbooks: Source of myths and misconceptions?. En R. Davidson \& J. Swift (Eds.), Proceedings of the Second International Conference on Teaching Statistics (pp. 127-131). Saanich: University of Victoria.

Brousseau, G. (1976). Les obstacles épistémologiques et les problèmes en mathématiques. Recherches en Didactique des Mathématiques, 4(2), 165-198.

Brousseau, G. (1991). Théorie des situations didactiques. Grenoble: La Pensée Sauvage.

Cabero, J., Duarte, A., \& Barroso, J. (1989). La formación del profesorado en nuevas tecnologías: retos hacia el futuro. En J. Ferrés \& P. Marqués (Eds.), Comunicación educativa y nuevas tecnologías. Barcelona: Praxis.

Cabero, J., Duarte, A., \& Romero, R. (1995). Los libros de texto y sus potencialidades para el aprendizaje. En J. Cabero \& L. M. Villar (Eds.), Aspectos Críticos de una Refoma Educativa. Sevilla: Universidad de Sevilla. Secretariado de Publicaciones.

Campanario, J. M. (2001). ¿Qué puede hacer un profesor como tú o un alumno como el tuyo con un libro de texto como éste? Una relación de actividades poco convencionales. Enseñanza de las Ciencias: revista de investigación y experiencias didácticas, 19(3), 351364.

Cockcroft, W. H. (1985). Las matemáticas sí cuentan: informe Cockroft. Madrid, España: Ministerio de Educación y Ciencia, Subdirección General de Perfeccionamiento del Profesorado. 
Çokadar, H., \& Şahin, A. (2009). Prospective Elementary Science Teachers' Experiences with Offered Textbooks in Teacher Education. Dicle Üniversitesi Ziya Gökalp Eğitim Fakültesi Dergisi, 12, 114-127.

Choppin, A. (1992). Les manuels scolaires: historie et actualite. París: Hachette Education.

Choppin, A. (2000). Pasado y presente de los manuales escolares. En J. Ruiz Berrio (Ed.), La cultura escolar de Europa. Tendencias históricas emergentes (pp. 107-165). Madrid: Editorial Biblioteca Nueva.

Daniels, H., \& Zemelman, S. (2004). Out with textbooks, in with learning. Educational leadership, 14(1), 36-40.

Escolano Vizcarra, R., \& Gairín Sallán, J. M. (2005). Modelos de medida para la enseñanza del número racional en Educación Primaria. Unión. Revista Iberoamericana de Educación Matemática(1), 17-35.

Fernández Bravo, J. A. (2010). La resolución de problemas matemáticos. Creatividad y razonamiento en la mente de los niños. Madrid: Grupo Mayéutica Educación.

Fernández Palop, P. y Caballero García, P.A. (2013). El error matemático en los libros de texto de educación primaria. Comunicación presentada al I Congreso Nacional "Avances en Investigación Científica. Madrid, 11 de julio.

Fernández Palop, P., Caballero García, P. A., \& Fernández Bravo, J. A. (2013). ¿Yerra el niño o yerra el libro de yerra el libro de matemáticas? Números, 83(julio), 131-148.

Feynman, R. P. (1987). ¿Está Ud. de broma, Sr. Feynman?. Madrid: Alianza Editorial S.A.

Gallardo-Gil, M., Fernández-Navas, M., Sepúlveda-Ruiz, M. P., Serván, M. J., Yus, R., \& Barquín, J. (2010). Pisa y la Competencia Científica: un análisis de las pruebas de Pisa en el área de Ciencias. Relieve: Revista ELectrónica de Investigación y EValuación Educativa, 16(2). Consultado el 20 de septiembre de 2014, en http://www.uv.es/RELIEVE/v16n2/RELIEVEv16n2_6.htm

García Mateos, A., \& Caballero García, P. A. (2005). La tecnología digital en el aula: un instrumento al servicio de los procesos de enseñanza-aprendizaje. Madrid: Universidad Camilo José Cela.

Gauld, C. (1997). It Must Be True-It's in the Textbook! Australian Science Teachers Journal, 43(2), 21-26.

Ginsburg, A., Leinwand, S., Anstrom, T., \& Pollock, E. (2005). What the United States Can Learn From Singapore's World-Class Mathematics System. Washington: American Institutes for Research.

Haggarty, L., \& Pepin, B. (2002). An investigation of mathematics textbooks and their use in English, French and German classrooms: who gets an opportunity to learn what?. British Educational Research Journal, 28(4), 567-590.

Hamilton, D. (1990). What is a textbook?. Paradigm, 3(Julio), 5-8.

Hatzinikita, V., Dimopoulos, K., \& Christidou, K. (2008). PISA test items and school textbooks related to science: A textual comparison. Science Education, 92 (4), 664 687.

Hubisz, J. (2001). Popular science textbooks found prone to error. Curriculum Review, 40(8), $1-5$. 
Instituto de Evaluación del Ministerio de Educación. (2009). Sistema estatal de indicadores de la educación. Madrid: Catálogo de Publicaciones del Ministerio: educación.es.

Jaime Pastor, A., Chapa Aguilera, F., \& Gutiérrez Rodríguez, A. (1992). Definiciones de triángulos y cuadriláteros: errores e inconsistencias en libros de texto de E.G.B. Epsilon, 23, 49-62.

Johnsen, E. B. (1996). Libros de texto en el caleidoscopio. Barcelona: Pomares-Corredor S.A.

Kimberley, P., Norris, S. P., Phillips, L. M., \& Clarck, G. (2003). The anatomy of junior high school science textbooks: An analysis of textual characteristics and a comparison to media reports of science. Canadian Journal of Science, Mathematics and Technology Education, 3(4), 415-436.

Kline, M. (1973). Why Johnny Can't Add. Nueva York: Vintage Books/St. Martin's Press.

Kolmogorov, A. N. (1946). On the proof of the method of least squares. Uspehi Matem. Nauk, 57-70.

López Varona, J. A., \& Moreno Martínez, M. L. (1997). Resultados de Matemáticas. Tercer Estudio Internacional de Matemáticas y Ciencias. Ministerio de Educación y Cultura. Instituto Nacional de Calidad y Evaluación. Consultado el 14 de febrero de 2013 en http://www.educacion.gob.es/dctm/ievaluacion/internacional/timssmat.pdf?docum entld=0901e72b80110725

Ministerio de Educación. (2010). PISA 2009. Informe Español. Consultado el 14 de febrero de 2013, en http://www.educacion.gob.es/dctm/ministerio/horizontales/prensa/notas/2010/2010 1207-pisa2009-informe-espanol.pdf?documentld=0901e72b806ea35a

Ministerio de Educación Cultura y Deporte. (2012). PIRLS-TIMSS 2011. Estudio Internacional de progreso en comprensión lectora, matemáticas y ciencias. Volumen I: Informe Español. Consultado el 22 de febrero de 2013. en http://www.mecd.gob.es/dctm/inee/internacional/pirlstimss2011vol1.pdf?documentl $\mathrm{d}=0901 \mathrm{e} 72 \mathrm{~b} 8146 \mathrm{foca}$

Ministerio de Educación, Cultura y Deporte y el Observatorio de la Lectura y el Libro (2015). Informe sobre "El sector del libro en España 2013-2015". Recuperado en http://www.mecd.gob.es/dms/mecd/cultura-mecd/areascultura/libro/mc/observatoriolect/redirige/estudios-e-informes/elaborados-por-elobservatoriolect/sector-libro-abril2015/sector-libro-abril2015.pdf

Ministerio de Educación y Ciencia. (2004). Evaluación PISA 2003. Resumen de los primeros resultados en España. Consultado el 14 de febrero de 2013, en http://www.educacion.gob.es/dctm/ievaluacion/internacional/pisa2003resumenesp ana.pdf?documentld=0901e $72 \mathrm{~b} 80110700$

Ministerio de Educación y Ciencia. (2007). PISA 2006. Informe Español. Consultado el 14 de febrero de 2013, en http://www.mec.es/multimedia/00005713.pdf

Ministerio de Educación y Ciencia. (2008). PISA 2003. Matemáticas. Informe Español. Consultado el 14 de febrero de 2013, en http://www.educacion.gob.es/dctm/ievaluacion/internacional/pisa2003mat.pdf?doc umentld=0901e72b80110553

Ministerio de Educación y Política Social y Deporte. (2005). Resultados en España del estudio PISA 2000. Consultado el 14 de febrero de 2013, en 
http://www.educacion.gob.es/dctm/ievaluacion/internacional/pisa200oinfnacional.p df?documentld=0901e72b8011069b

Ministry of Education. (2011). Primary Mathematics (Standards Edition) Textbook 1-5, A-B. Singapore: Marshall Cavendish Education.

Moya Pardo, C. (2008). Aproximación al concepto y tratamiento de texto escolar. Cuadernos de Ligüística Hispánica, 11(Enero-Junio), 133-152.

Mullis, I. V. S., Martin, M. M., Foy, P., \& Arora, A. (2012). TIMSS 2011 International. Results in Mathematics. Boston: TIMSS \& PIRLS International Study Center.

Muntean, A. (2011). Sobre algunos errores en libros de texto de Matemáticas de Secundaria y Bachillerato. Boletín (Sociedad Puig Adam de Profesores de Matemáticas), 87, 30-53.

OECD. (2012). PISA en español. OCDE Programme for International Student Assessment (PISA). Consultado el 1 de marzo de 2013, en http://www.oecd.org/document/25/0,3746,en_32252351_32235731_39733465_1_1_1_ 1,00.html

Orlans, H. (1998). It must be true. Change, 30(4), 11.

Parcerisa Aran, A. (1996). Materiales curriculares: cómo elaborarlos, seleccionarlos y usarlos (Vol. 105). Barcelona, España: Grao.

Prendes, M. P. (1994). La imagen didáctica: análisis descriptivo y evaluativo. Tesis Doctoral no publicada. Murcia: Universidad de Murcia.

Prendes, M. P. (2001). Evaluación de manuales escolares. Pixel-Bit: Revista de medios y educación, 16.

Puget, M. (1963). Le livre scolaire. Tendances, 22(avril), 212-230.

Richaudeau, F. (1981). Concepción y producción de manuales escolares. París: Serbal/UNESCO.

Romero, N.E. (2011). Entre maestros y editores: Sentidos atribuidos a las prácticas escolares asociadas al uso de los textos en escuelas primarias de la provincia y la ciudad de Buenos Aires. Tesis doctoral. Buenos Aires: Universidad de San Andrés.

Roseman, J. E., Kulm, G., \& Shuttleworth, S. (2001). Putting textbooks to the test. ENC Focus, 8(3), 56-59.

Stern, L., \& Roseman, J. E. (2004). Can Middle-School Science Textbooks Help Students Learn Important Ideas? Findings from Project 2061's Curriculum Evaluation Study: Life Science. Journal of Research in Science Teaching, 41(6), 538-568.

The Georg Eckert Institute for International Textbook Research (2016). The Institute. Recuperado en http://www.gei.de/en/the-institute.html

Torres Santomé, J. (1994). Globalización e interdisciplinariedad: el curriculum integrado. Madrid, España: Morata.

Varela Mallou, J. (2008). El Libro de Texto ante la Incorporación de las TIC a la Enseñanza. Santiago de Compostela, España: Universidad de Santiago de Compostela.

Venegas, M. C. (1993). El texto escolar: cómo aprovecharlo. Santafé de Bogotá: Ministerio de Educación Nacional.

Wilkinson, J. (1999). A quantitative analysis of physics textbooks for scientific literacy themes. Research in Science Education, 29(3), 385-399. 